\title{
]jfis
}

\section{On the Left and Right Almost Hyperideals of LA-Semihypergroups}

\author{
Shah Nawaz ${ }^{1}$, Muhammad Gulistan ${ }^{1}$, Nasreen Kausar ${ }^{2}$, Salahuddin ${ }^{3}$, and \\ Mohammad Munir ${ }^{4}$ \\ ${ }^{1}$ Department of Mathematics and Statistics, Hazara University, Mansehra, Pakistan \\ ${ }^{2}$ Department of Mathematics and Statistics, University of Agriculture, Faisalabad, Pakistan \\ ${ }^{3}$ Department of Mathematics, Jazan University, Jazan, Kingdom of Saudi Arabia \\ ${ }^{4}$ Department of Mathematics, Government Postgraduate College, Abbottabad, Pakistan
}

Received: Dec. 17, 2020

Revised : Jan. 29, 2021

Accepted: Feb. 22, 2021

Correspondence to: Nasreen Kausar (kausar.nasreen57@gmail.com)

@The Korean Institute of Intelligent Systems

cc) This is an Open Access article distributed under the terms of the Creative Commons Attribution Non-Commercial License (http://creativecommons.org/licenses/by-nc/ 3.0/) which permits unrestricted noncommercial use, distribution, and reproduction in any medium, provided the original work is properly cited.

\begin{abstract}
In this paper, we define left almost hyperideals, right almost hyperideals, almost hyperideals, and minimal almost hyperideals. We demonstrate that the intersection of almost hyperideals is not required to be an almost hyperideal, but the union of almost hyperideals is an almost hyperideal, which is completely different from the classical algebraic concept of the ideal theory.
\end{abstract}

Keywords: LA-semihypergroups, Left almost hyperideals, Right almost hyperideals, Almost hyperideals, Minimal almost hyperideals

\section{Introduction}

Hyperstructures were introduced in 1934 when Marty [1] defined hypergroups, began to study their properties, and applied them to groups. Several papers and books have been written on hyperstructure theory [2]3]. Currently, a book published on hyperstructures [4] describes its applications in rough set theory, cryptography, automata, code automata, probability, geometry, lattices, binary relations, graphs, and hypergraphs.

Kazim and Naseeruddin [5] introduced the study of left almost semigroups (LAsemigroups). They generalized some useful sequels of semigroup theory. Subsequently, Mushtaq and his colleagues [6-13] further described the structure and included many useful results of theory of an LA-semigroup [7|14-20]. An LA-semigroup is the midway structure between a commutative semigroup and a groupoid. Moreover, it has many interesting properties, which are frequently used in commutative and associative algebraic structures.

Hila and Dine [21] presented the concept of LA-semihypergroups. Their paper created a new research direction in non-associative hyperstructures. Yaqoob et al. [22] extended the work of Hila and Dine [21] and characterized intra-regular LA-semihypergroups by their hyperideals using pure left identities. Subsequently, many researchers studied the structures of LA-semihypergroups from different perspectives [23-30].

Azhar and his colleagues [31-34] developed the structures of partially ordered LAsemihypergroups. Nawaz and his colleagues [35-41] developed and studied many nonassociative structures. The concept of almost ideals in semigroups was provided by Grosek and Satko [42 43] in 1980 and 1981, respectively. Various other researchers studied almost ideals for different algebraic structures [44-46]. 
Recently, for the first time, Suebsung et. al [48] developed the concept of almost hyperideals in semihypergroups (see also [48|49]).

Thus, inspired by the concept by Suebsung et. al [47], we developed structures of almost hyperideals in LA-semihypergroups. These new types of almost hyperideals have two main characteristics. First, they are purely non-associative substructures of LA-semihypergroups. Second, in the construction of these hyperideals, we ensured that a hyperoperation did not yield a single element as in the scenario of the paper by Suebsung et. al [47]. We proved some useful results related to almost hyperideals.

\section{LA-Semihypergroups}

In this section, we recall some basic concepts from the literature on ideals and LA-semihypergroups that were used in the further development of this article.

Definition 1. A map $\circ: X \times X \longrightarrow \mathcal{P}^{*}(X)$ is called an hyperoperation or join operation on the set $X$, where $X$ is a non-empty set and $\mathcal{P}^{*}(X)=\mathcal{P}(X) \backslash\{\emptyset\}$ indicates the all nonempty subsets of $X$. A hypergroupoid is a set $(X)$ for which the binary operation is a hyperoperation.

If $A$ and $B$ are two non-empty subsets of $X$, then we express the product as follows:

$$
\begin{aligned}
& A \circ B=\bigcup_{x_{1} \in A, x_{2} \in B} x_{1} \circ x_{2}, x_{1} \circ A=\left\{x_{1}\right\} \circ A \\
& \text { and } x_{1} \circ B=\left\{x_{1}\right\} \circ B .
\end{aligned}
$$

Definition 2 ([21 22] ). A hypergroupoid $(X, \circ)$ is called an LA-semihypergroup if for $x_{1}, x_{2}, x_{3} \in X$,

$$
\left(x_{1} \circ x_{2}\right) \circ x_{3}=\left(x_{3} \circ x_{2}\right) \circ x_{1} .
$$

The law $\left(x_{1} \circ x_{2}\right) \circ x_{3}=\left(x_{3} \circ x_{2}\right) \circ x_{1}$ is known as left invertive law.

Every LA-semihypergroup satisfies the law

$$
\left(x_{1} \circ x_{2}\right) \circ\left(x_{3} \circ x_{4}\right)=\left(x_{1} \circ x_{3}\right) \circ\left(x_{2} \circ x_{4}\right)
$$

for all $x_{1}, x_{2}, x_{3}, x_{4} \in X$. This law is known as medial law (cf. [21]).

Definition 3. [22] Let $X$ be an LA-semihypergroup. Element $e \in X$ is called a
1. Left identity (resp. pure left identity) if for all $x_{1} \in X$, $x_{1} \in e \circ x_{1}$ (resp. $x_{1}=e \circ x_{1}$ ),

2. Right identity (resp. pure right identity) if for all $x_{1} \in X$, $x_{1} \in x_{1} \circ e\left(\right.$ resp. $\left.z_{1}=z_{1} \circ e\right)$,

3. Identity (resp. pure identity) if for all $z_{1} \in H, z_{1} \in$ $e \circ z_{1} \cap z_{1} \circ e$ (resp. $\left.x_{1}=e \circ z_{1} \cap z_{1} \circ e\right)$.

Example 1 ([22]). Let $X=\left\{l_{1}, l_{2}, l_{3}, l_{4}\right\}$ with the binary hyperoperation defined as follows:

\begin{tabular}{|c|c|c|c|c|}
\hline$\circ$ & $l_{1}$ & $l_{2}$ & $l_{3}$ & $l_{4}$ \\
\hline$l_{1}$ & $l_{1}$ & $l_{2}$ & $l_{3}$ & $l_{4}$ \\
\hline$l_{2}$ & $l_{3}$ & $\left\{l_{2}, l_{3}\right\}$ & $\left\{l_{2}, l_{3}\right\}$ & $l_{4}$ \\
\hline$l_{3}$ & $l_{2}$ & $\left\{l_{2}, l_{3}\right\}$ & $\left\{l_{2}, l_{3}\right\}$ & $l_{4}$ \\
\hline$l_{4}$ & $l_{4}$ & $l_{4}$ & $l_{4}$ & $H$ \\
\hline
\end{tabular}

Hence $X$ is an LA-semihypergroup because it satisfies the left invertive law. AS the table shows, $l_{1}$ is a left identity of $X$.

Lemma 1 ([22]). Let $X$ be an LA-semihypergroup with pure left identity $e$; then $x_{1} \circ\left(x_{2} \circ x_{3}\right)=x_{2} \circ\left(x_{1} \circ x_{3}\right)$ holds for all $x_{1}, x_{2}, x_{3} \in X$.

Lemma 2 ([22]). Let $X$ be an LA-semihypergroup with pure left identity $e$; then $\left(x_{1} \circ x_{2}\right) \circ\left(x_{3} \circ x_{4}\right)=\left(x_{4} \circ x_{2}\right) \circ\left(x_{3} \circ x_{1}\right)$ holds for all $x_{1}, x_{2}, x_{3}, x_{4} \in X$.

The law $\left(x_{1} \circ x_{2}\right) \circ\left(x_{3} \circ x_{4}\right)=\left(x_{4} \circ x_{2}\right) \circ\left(x_{3} \circ x_{1}\right)$ is called a paramedial law.

Definition 4 ([47|). Let $(H, \circ)$ be a semihypergroup.

(1) A non-empty subset $L$ of $H$ is called the left almost hyperideal of $H$ if

$$
x \circ L \cap L \neq \phi, \forall x \in H \text {. }
$$

(2) A non-empty subset $L$ of $H$ is called the right almost hyperideal of $H$ if

$$
L \circ x \cap L \neq \phi, \forall x \in H \text {. }
$$

(3) A non-empty subset $L$ of $H$ is called the almost hyperideal of $H$ if $L$ is both a left and right almost hyperideal of $H$.

\section{Almost Hyperideals}

Here we define left almost hyperideals, right almost hyperideals, almost hyperideals, minimal almost hyperideals, and some interesting properties. 
Definition 5. Let $(H, *)$ be an LA-semihypergroup.

(1) A non-empty subset $L$ of $H$ is called the left almost hyperideal of $H$ if

$$
x * L \cap L \neq \phi, \forall x \in H .
$$

(2) A non-empty subset $R$ of $H$ is called the right almost hyperideal of $H$ if

$$
R * x \cap R \neq \phi, \forall x \in H .
$$

(3) A non-empty subset $I$ of $H$ is called the almost hyperideal of $H$ if $I$ is both a left and right almost hyperideal of $H$.

Example 2. Let $H=\{e, l, m\}$ with the binary operation * defined as follows:

\begin{tabular}{|c|c|c|c|}
\hline$*$ & $e$ & $l$ & $m$ \\
\hline$e$ & $e$ & $l$ & $m$ \\
\hline$l$ & $m$ & $\{l, m\}$ & $m$ \\
\hline$m$ & $l$ & $l$ & $\{l, m\}$ \\
\hline
\end{tabular}

$(H, *)$ is an LA-semihypergroup. Now, if $L=\{e, l\} \subseteq H$ with

\begin{tabular}{|c|c|c|}
\hline$*$ & $e$ & $l$ \\
\hline$e$ & $e$ & $l$ \\
\hline$l$ & $m$ & $\{l, m\}$ \\
\hline
\end{tabular}

we observe that $(L, *)$ is a left almost hyperideal of $(H, *)$, but it is not a right almost hyperideal as $\{e, l\} * m \cap\{e, l\}=\phi$.

Remark 1. The structure of left almost hyperideals is not LAsubsemihypergroups as in Example 2, where $L=\{e, l\} \subseteq H$ is not closed under the same binary operation as $H$.

Example 3. Consider an LA-semihypergroup $H=\{a, b, c\}$ under the hyperoperation defined by

\begin{tabular}{|c|c|c|c|}
\hline$\circ$ & $a$ & $b$ & $c$ \\
\hline$a$ & $a$ & $b$ & $c$ \\
\hline$b$ & $c$ & $\{b, c\}$ & $\{b, c\}$ \\
\hline$c$ & $b$ & $\{b, c\}$ & $\{b, c\}$ \\
\hline
\end{tabular}

If $R=\{b, c\} \subseteq H$ with the same binary operation $\circ$ that is defined in $H$, we can observe that $R$ is a right almost hyperideal of $H$.

Lemma 3. Let $(H, *)$ be a LA-semihypergroup.

(1) Every left hyperideal of $H$ is a left almost hyperideal of $H$.
(2) Every right hyperideal of $H$ is a left almost hyperideal of $H$.

(3) Every hyperideal of $H$ is an almost hyperideal of $H$.

Proof. (1) Assume that $L$ is a left hyperideal of $H$. Let $x \in H$. Then, $x * L \subseteq L$. Therefore, $x * L \cap L \neq \phi$.

(2) Let $R$ be a right hyperideal of $H$. Let $y \in H$. Then, $R * y \subseteq R$. Therefore, $R * y \cap R \neq \phi$.

(3) Follows from (1) and (2)

Remark 2. The converse of Lemma 3 may or may not true generally as in the following Example 4

Example 4. Let $H=\{x, y, z\}$ be a finite set with hyperoperation $*$ defined as follows:

\begin{tabular}{|c|c|c|c|}
\hline$*$ & $x$ & $y$ & $z$ \\
\hline$x$ & $x$ & $x$ & $x$ \\
\hline$y$ & $x$ & $H$ & $z$ \\
\hline$z$ & $x$ & $H$ & $H$ \\
\hline
\end{tabular}

Thus, $(H, *)$ is an LA-semihypergroup. Let $L=\{x, y\}$ under the same binary operation $*$, i.e.,

\begin{tabular}{|c|c|c|}
\hline$*$ & $x$ & $y$ \\
\hline$x$ & $x$ & $x$ \\
\hline$y$ & $x$ & $H$ \\
\hline
\end{tabular}

Here, $L$ is an almost hyperideal of $H$, but it is not a hyperideal of $H$.

Theorem 1. Let $(H, *)$ be an LA-semihypergroup.

(1) If $L_{1}$ and $L_{2}$ are two left almost hyperideals of $H$, then $L_{1} \cup L_{2}$ is also a left almost hyperideal.

(2) If $R_{1}$ and $R_{2}$ are two right almost hyperideals of $H$, then $R_{1} \cup R_{2}$ is also a right almost hyperideal.

(3) If $I_{1}$ and $I_{2}$ are two almost hyperideals of $H$, then $I_{1} \cup I_{2}$ is also an almost hyperideal.

Proof. Same as provided by Suebsung et. al [47].

Remark 3. (1) If $L_{1}$ and $L_{2}$ are two left almost hyperideals of $H$, then $L_{1} \cap L_{2}$ may or may not a left almost hyperideal.

(2) If $R_{1}$ and $R_{2}$ are two right almost hyperideals of $H$, then $R \cap R_{2}$ may or may not a right almost hyperideal.

(3) If $I_{1}$ and $I_{2}$ are two almost hyperideals of $H$, then $I_{1} \cap I_{2}$ may or may not left almost hyperideal.

Example 5. Let $H=\{1,2,3,4,5,6\}$ be a non-empty set with hyperoperation $*$, defined as follows: then $(H, *)$ is an LA- 


\begin{tabular}{|c|c|c|c|c|c|c|}
\hline$*$ & 1 & 2 & 3 & 4 & 5 & 6 \\
\hline 1 & $\{1,2,3,4\}$ & $\{2,3,4,5,6\}$ & $\{4,5,6\}$ & $\{2,3,4,5,6\}$ & $\{2,3,4,5,6\}$ & $\{2,3,4,5,6\}$ \\
\hline 2 & $\{1,3,4,5,6\}$ & $\{1,2,3,4,5,6\}$ & $\{1,3,4,5,6\}$ & $\{1,3,6\}$ & $\{1,5,6\}$ & $\{1,3,4,5,6\}$ \\
\hline 3 & $\{1,2,4,5,6\}$ & $\{1,2\}$ & $\{1,2,3,5,6\}$ & $\{1,2,4,5,6\}$ & $\{1,2,6\}$ & $\{1,2,4,5,6\}$ \\
\hline 4 & $\{1,5,6\}$ & $\{1,2,6\}$ & $\{1,2,5,6\}$ & $\{1,2,5,6\}$ & $\{1,2,5,6\}$ & $\{1,2,5,6\}$ \\
\hline 5 & $\{1,2,3,4,6\}$ & $\{1,2,3,4,6\}$ & $\{1,2,3,4,6\}$ & $\{1,3,4,6\}$ & $\{1,2,3,4,5,6\}$ & $\{1,3,4,6\}$ \\
\hline 6 & $\{1,2,3,4,5\}$ & $\{1,4,5\}$ & $\{1,2,3,4,5\}$ & $\{1,4,5\}$ & $\{1,2,3,4,5\}$ & $\{1,2,3,4,5\}$ \\
\hline
\end{tabular}

semihypergroup. Let $L_{1}=\{1,2,3,4\}$ and $L_{2}=\{3,4,5,6\}$ be two left almost hyperideals of $H$. Thus, $L_{1} \cap L_{2}=\{3,4\}$ is not a left almost hyperideal of $H$, as we can observe that $4 *\{3,4\} \cap\{3,4\}=\phi$. However, $L_{1} \cup L_{2}=\{1,2,3,4,5,6\}$ is a left almost hyperideal of $H$.

Lemma 4. If $L$ is a left almost hyperideal of an LA-semihypergroup $H$ with a left identity $e$; then, $a L$ is a left almost hyperideal of $H$.

Proof. However, if $a L$ is not a left almost hyperideal, i.e.,

$$
\begin{aligned}
x * a L \cap a L & =\phi \text { for all } x \in H \\
& \Rightarrow a * x L \cap a L \\
& =\phi, \text { by }(a b) c=b(a c) \\
& \Rightarrow x L \cap L=\phi,
\end{aligned}
$$

this is a contradiction to the given condition. Hence, $x * a L \cap$ $a L \neq \phi$ and $a L$ is a left almost hyperideal of $H$.

Lemma 5. If $L$ is a left almost hyperideal of an LA-semihypergroup $H$ with a left identity $e$, then $L^{2}$ is a left almost hyperideal of $H$.

Proof. However, if $L^{2}$ is not a left almost hyperideal, i.e.,

$$
\begin{aligned}
x * L^{2} \cap L^{2} & =\phi \text { for all } x \in H, \\
& \Rightarrow x * L * L \cap L * L \\
& \Rightarrow(x * L) * L \cap L * L \\
& \Rightarrow L *(x * L) \cap L * L=\phi, \text { by }(a b) c=b(a c) \\
& \Rightarrow x L \cap L=\phi,
\end{aligned}
$$

this is a contradiction to the given condition. Hence, $x * L^{2} \cap$ $L^{2} \neq \phi$ and $L^{2}$ is a left almost hyperideal of $H$.

Lemma 6. If $I$ is a left almost hyperideal of $H$, then $I^{2}$ is an almost hyperideal of $H$.

Proof. First, we must demonstrate that $I^{2}$ is a left-almost hyperideal of $H$. This, we demonstrate that $x * I^{2} \cap I^{2} \neq \phi \Rightarrow$ $x * I * I \cap I * I$, as $x * I \cap I \neq \phi$. Hence $x * I^{2} \cap I^{2} \neq \phi$ Therefore,
$I^{2}$ is a left almost ideal of $H$. Next, we must demonstrate that $I^{2} * x \cap I^{2} \neq \phi \Rightarrow(I * I) * x \cap I * I$, as $(x * I) * I \cap I * I \neq$ by $(a b) c=(c b) a$. As $x * I \cap I \neq \phi, I^{2} * x \cap I^{2} \neq \phi$. Therefore, $I^{2}$ is a right almost hyperideal of $H$. Hence, $I^{2}$ is an almost hyperideal of $H$.

Definition 6. An almost hyperideal $I$ of an LA-semihypergroup $H$ is minimal if it does not contain any almost hyperideal of $H$ other than itself.

Example 6. Let $H=\left\{x_{1}, x_{2}, x_{3}, x_{4}\right\}$ with the binary hyperoperation defined as follows:

\begin{tabular}{|c|c|c|c|c|}
\hline$\circ$ & $x_{1}$ & $x_{2}$ & $x_{3}$ & $x_{4}$ \\
\hline$x_{1}$ & $x_{1}$ & $x_{2}$ & $x_{3}$ & $x_{4}$ \\
\hline$x_{2}$ & $x_{3}$ & $\left\{x_{2}, x_{3}\right\}$ & $\left\{x_{2}, x_{3}\right\}$ & $x_{4}$ \\
\hline$x_{3}$ & $x_{2}$ & $\left\{x_{2}, x_{3}\right\}$ & $\left\{x_{2}, x_{3}\right\}$ & $x_{4}$ \\
\hline$x_{4}$ & $x_{4}$ & $x_{4}$ & $x_{4}$ & $H$ \\
\hline
\end{tabular}

Hence $H$ is an LA-semihypergroup. Let $I_{1}=\left\{x_{1}, x_{2}, x_{4}\right\}$, $I_{2}=\left\{x_{1}, x_{3}, x_{4}\right\}$ and $I_{3}=\left\{x_{4}\right\}$ are the almost hyperideals of $H$. Thus, $I_{3}$ is a minimal almost hyperideal of $H$.

Theorem 2. Let $H$ be an LA-semihypergroup. If $L$ is a minimal almost left hyperideal of $H$, then $a * L$ is also a minimal almost left hyperideal of $H$ for every weak idempotent $a$.

Proof. It is known that $a * L$ is a left almost hyperideal of $H$. Next, we must demonstrate that $a * L$ is minimal almost left hyperideal of $H$. Let $L$ be a left almost hyperideal of $H$, which is properly contained in $a * L$. We define $K=\{i \in I: a * i \subseteq L\}$ and let $y \in K$. Let $a * i \in L \Longrightarrow x *(a * i) \cap a * i \neq \phi \Longrightarrow$ $x * L \cap L \neq \phi \Longrightarrow a * i \in L$, which is contradiction to the minimality of $L$. Therefore, $a * L$ is minimal almost left hyperideal.

\section{Conclusion}

In this paper, we have introduced new types of hyperideals in non-associative structures of semihypergroups and indicated some interesting properties. In the future, we aim to obtain more properties of the proposed ideal theory. 


\section{Conflict of Interest}

No potential conflict of interest relevant to this article was reported.

\section{References}

[1] F. Marty, "Sur une generalization de la notion de groupe," in Proceedings of the 8th Congress des Mathematicians Scandinaves, Stockholm, Sweden, 1934, pp. 45-49.

[2] P. Corsini, Prolegomena of Hypergroup Theory. Tricesimo, Italy: Aviani Editore, 1993.

[3] T. Vougiouklis, Hyperstructures and Their Representations. Palm Harbor, FL: Hadronic Press, 1994.

[4] P. Corsini and V. Leoreanu, Applications of Hyperstructure Theory. Boston, MA: Springer, 2003.

[5] M. A. Kazim and M. D. Naseeruddin, "On almost semigroups," The Aligarh Bulletin of Mathematics, vol. 2, pp. $1-7,1972$.

[6] Q. Mushtaq and S. M. Yusuf, "On LA-semigroups," The Aligarh Bulletin of Mathematics, vol. 8, pp. 65-70, 1978.

[7] Q. Mushtaq and S. M. Yousaf, “On locally associative LA-semigroups," Journal of Natural Sciences and Mathematics, vol. 19, no. 1, pp. 57-62, 1979.

[8] Q. Mushtaq, M. Khan, and K. P. Shum, "Topological structures on LA-semigroups," Bulletin of the Malaysian Mathematical Sciences Society, vol. 36, no. 4, pp. 901-906, 2013.

[9] Q. Mushtaq, “A note on almost semigroups," Bulletin of the Malaysian Mathematical Sciences Society, vol. 11, no. 2, pp. 29-31, 1988.

[10] Q. Mushtaq, "A note on translative mappings on LAsemigroups," ulletin of the Malaysian Mathematical Sciences Society, vol. 11, no. 2, pp. 39-42, 1988.

[11] Q. Mushtaq and M. Inam, "Left almost semigroups defined by a free algebra," Quasigroups and Related Systems, vol. 16, no. 1, 69-76. 2008.

[12] Q. Mushtaq, "Abelian group defined by LA-semigroup," Studia Scientiarum Mathematicarum Hungarica, vol. 18, pp. 427-428, 1983.
[13] Q. Mushtaq and M. S. Kamran, “On left almost groups,' Proceedings of the Pakistan Academy of Sciences, vol. 33, no. 1, pp. 53-55, 1996.

[14] P. Holgate, "Groupoids satisfying a simple invertive law," Mathematics Student, vol. 61, no. 1-4, pp. 101-106, 1992.

[15] J. R. Cho, J. Jezek, and T. Kepka, "Paramedial groupoids," Czechoslovak Mathematical Journal, vol. 49, no. 2, pp. 277-290, 1999.

[16] M. Akram, N. Yaqoob, and M. Khan, “On (m, n)-ideals in LA-semigroups," Applied Mathematical Sciences, vol. 7, no. 44, pp. 2187-2191, 2013.

[17] M. Khan and N. Ahmad, "Characterizations of left almost semigroups by their ideals," Journal of Advanced Research in Pure Mathematics, vol. 2, no. 3, pp. 61-73, 2010.

[18] P. V. Protic and N. Stevanovic, "AG-test and some general properties of Abel-Grassmann's groupoids," Pure Mathematics and Applications, vol. 6, no. 4, pp. 371-383, 1995.

[19] N. Stevanovic and P. V. Protic, "Composition of AbelGrassmann's three 3-bands," Novi Sad Journal of Mathematics, vol. 34, no. 2, pp. 175-182, 2004.

[20] M. Iqbal and I. Ahmad, "On further study of CA-AGgroupoids," Proceedings of the Pakistan Academy of Sciences A: Physical and Computational Sciences, vol. 53, no. 3, pp. 325-337, 2016.

[21] K. Hila and J. Dine, "On hyperideals in left almost semihypergroups," International Scholarly Research Notices, vol 2011, article no. 953124, 2011. http://doi.org/10.5402/ $2011 / 953124$

[22] N. Yaqoob, P. Corsini, and F. Yousafzai, “On intra-regular left almost semihypergroups with pure left identity," Journal of Mathematics, vol., 2013, article no. 510790, 2013. https://doi.org/10.1155/2013/510790

[23] N. Yaqoob and M. Gulistan, "Partially ordered left almost semihypergroups," Journal of the Egyptian Mathematical Society, vol. 23, no. 2, pp. 231-235, 2015. https://doi.org/ 10.1016/j.joems.2014.05.012

[24] M. Gulistan, N. Yaqoob, and M. Shahzad, "A note on Hv-LA-semigroups," UPB Scientific Bulletin, vol. 77, no. 3, 93-106, 2015 
[25] M. Khan, M. Gulistan, N. Yaqoob, and F. Hussain, "General cubic hyperideals of LA-semihypergroups," Afrika Matematika, vol. 27, no. 3-4, 731-751, 2016. https://doi. org/10.1007/s13370-015-0367-y

[26] M. Gulistan, M. Khan, N. Yaqoob, and M. Shahzad, "Structural properties of cubic sets in regular LAsemihypergroups," Fuzzy Information and Engineering, vol. 9 no. 1, pp. $93-116,2017$. https://doi.org/10.1016/j. fiae.2017.03.005

[27] N. Yaqoob, M. Gulistan, V. Leoreanu-Fotea, and K. Hila, "Cubic hyperideals in LA-semihypergroups," Journal of Intelligent \& Fuzzy Systems, vol. 34, no. 4, pp. 2707-2721, 2018. https://doi.org/10.3233/JIFS-17850

[28] W. Nakkhasen, "Intuitionistic fuzzy ideals of ternary near-rings," International Journal of Fuzzy Logic and Intelligent Systems, vol. 20, no. 4, pp. 290-297, 2020. https://doi.org/10.5391/IJFIS.2020.20.4.290

[29] K. Hur, S. R. Kim, and P. K. Lim, "Intuitionistic fuzzy k-ideals of a semiring," International Journal of Fuzzy Logic and Intelligent Systems, vol. 9, no. 2, pp. 110-114, 2009. https://doi.org/10.5391/IJFIS.2009.9.2.110

[30] J. H. Park, "Operations on generalized intuitionistic fuzzy soft sets," International Journal of Fuzzy Logic and Intelligent Systems, vol. 11, no. 3, pp. 184-189, 2011. https://doi.org/10.5391/IJFIS.2011.11.3.184

[31] M. Azhar, M. Gulistan, N. Yaqoob, and S. Kadry, "On fuzzy ordered LA-semihypergroups," International Journal of Analysis and Applications, vol. 16, no. 2, pp. 276289, 2018. https://doi.org/10.28924/2291-8639-16-2018276

[32] M. Azhar, N. Yaqoob, M. Gulistan, and M. M. Khalaf, "On $\left(\varepsilon, \varepsilon \vee q_{k}\right)$-fuzzy hyperideals in ordered LAsemigroups," Discrete Dynamics in Nature and Society, vol. 2018, article no. 9494072, 2019. https://doi.org/10. 1155/2018/9494072

[33] M. Gulistan, N. Yaqoob, S. Kadry, and M. Azhar, "On generalized fuzzy sets in ordered LA-semihypergroups," Proceedings of the Estonian Academy of Sciences, vol. 68, no.1, pp. 43-54, 2019. https://doi.org/10.3176/proc.2019. 1.06
[34] N. Yaqoob, M. Gulistan, J. Tang, and M. Azhar, "On generalized fuzzy hyperideals in ordered LA-semihypergroups," Computational and Applied Mathematics, vol. 38, article no. 124, 2019. https://doi.org/10.1007/s40314-019-08767

[35] S. Nawaz, M. Gulistan, and S. Khan, "Weak LAhypergroups: neutrosophy, enumeration and redox reaction," Neutrosophic Sets and Systems, vol. 36, pp. 351-368, 2020.

[36] M. Gulistan, S. Nawaz, and N. Hassan, "Neutrosophic triplet non-associative semihypergroups with application," Symmetry, vol. 10, no. 11, article no. 613, 2018. https: //doi.org/10.3390/sym10110613

[37] I. Rehman, M. Gulistan, M. Asif Gondal, and S. Nawaz, "Structures of generalized fuzzy sets in non-associative rings," International Journal of Pure and Applied Mathematics, vol. 113, no. 2, 299-325, 2017.

[38] N. Yaqoob, I. Cristea, M. Gulistan, and S. Nawaz, "On left almost polygroups," Italian Journal of Pure and Applied Mathematics, vol. 39, pp. 465-474, 2018.

[39] S. Nawaz, I. Rehman, and M. Gulistan, "On left almost semihyperrings," International Journal of Analysis and Applications, vol. 16, no. 4, pp. 528-541, 2018. https: //doi.org/10.28924/2291-8639-16-2018-528

[40] M. Gulistan, N. Yaqoob, S. Nawaz, and M. Azhar, "A study of $(\alpha, \beta)$-complex fuzzy hyperideals in nonassociative hyperrings," Journal of Intelligent \& Fuzzy Systems, vol. 36, no. 6, pp. 6025-6036, 2019. https: //doi.org/10.3233/JIFS-181829

[41] S. Nawaz, M. Gulistan, N. Yaqoob, and S. Kadry, "Weak non-associative structures of groups with applications,' International Journal of Analysis and Applications, vol. 17, no. 5, pp. 864-878, 2019. https://doi.org/10.28924/ 2291-8639-17-2019-864

[42] O. Grosek, and L. Satko, "A new notion in the theory of semigroups," Semigroup Forum, vol. 20, pp. 233-240, 1980. https://doi.org/10.1007/BF02572683

[43] O. Grosek, and L. Satko, "Smallest A-ideals in semigroups," Semigroup Forum, vol. 23, pp. 297-309, 1981. https://doi.org/10.1007/BF02676654 
[44] K. Wattanatripop, R. Chinram, and T. Changphas, "QuasiA-ideals and fuzzy A-ideals in semigroups," Journal of Discrete Mathematical Sciences and Cryptography, vol. 21, no. 5, pp. 1131-1138, 2018. https://doi.org/10.1080/ 09720529.2018 .1468608

[45] K. Wattanatripop and T. Changphas, "On left and right A-ideals of a $\Gamma$-semigroup," Thai Journal of Mathematics, vol. 2018 Special Issue, pp. 87-96, 2018.

[46] S. Suebsung, K. Wattanatripop, and R. Chinram, "Aideals and fuzzy A-ideals of ternary semigroups," Songklanakarin Journal of Science \& Technology, vol. 41, no. 2, pp. 299-304, 2019.

[47] S. Suebusing, T. Kaewnoi, and R. Chinram, "A note on almost hyperideals in semihypergroups," International Journal of Mathematics and Computer Science, vol. 15, no. 1, pp. 127-133, 2020.

[48] T. C. Ahn, K. Hur, and K. W. Jang, "Intuitionistic fuzzy subgroups and level subgroups," International Journal of Fuzzy Logic and Intelligent Systems, vol. 6, no. 3, pp. 240246, 2006. http://dx.doi.org/10.5391/IJFIS.2006.6.3.240

[49] K. Hur, S. Y. Jang, and P. K. Lim, "Intuitionistic fuzzy semigroups," International Journal of Fuzzy Logic and Intelligent Systems, vol. 8, no. 3, pp. 207-219, 2008. http: //dx.doi.org/10.5391/IJFIS.2008.8.3.207

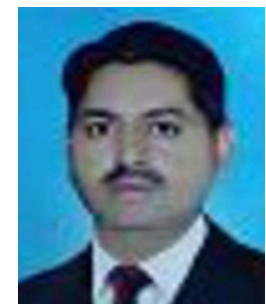

Shah Nawaz is a Ph.D. student of Dr. Muhammad Gulistan and has submitted his $\mathrm{Ph} . \mathrm{D}$. thesis in the field of non-associative hyperstructures. He has authored some papers on the introduction of new non-associative hyperstructures such as

\section{LA-polygroups and LA-hypergroups.}

E-mail: shahnawazawan82@gmail.com

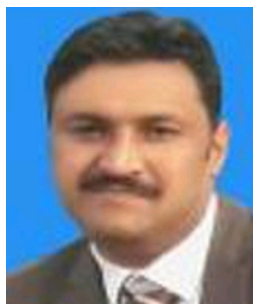

Muhammad Gulistan received his M.Phil degree from Quaid-i- Azam University, Islamabad, in 2011, and his Ph.D. degree from Hazara University, in 2016, where he is currently working as an Assistant Professor in the Department of Mathematics and Statistics. He has supervised many M.Phil. and Ph.D. research students. He has published more than 80 research papers in different well reputed journals. His area of research interests includes cubic sets and their generalizations, non-associative hyperstructures, neutrosophic cubic sets, neutrosophic cubic graphs, and decision making.

E-mail: gulistanmath@hu.edu.pk ORCID: https://orcid.org/0000-0002-6438-1047

Nasreen Kausar received her Ph.D. degree in Mathematics from the Quaid-i-Azam University in Islamabad, Pakistan. She is currently an assistant Professor of Mathematics at the University of Agriculture Faisalabad, Pakistan. Her research interests include the numerical analysis and numerical solutions of ordinary differential equations (ODEs), partial differential equations (PDEs), and Volterra integral equations. She also has research interests in associative and commutative, non-associative and non-commutative fuzzy algebraic structures and their applications. Department of Mathematics and Statistics University of Agriculture, Faisalabad, Pakistan.

E-mail: kausar.nasreen57@gmail.com

ORCID: https://orcid.org/0000-0002-8659-0747

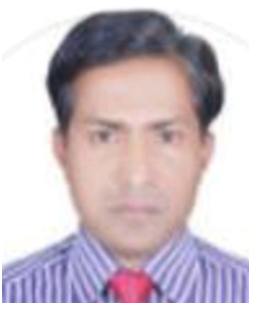

Salahuddin received his $\mathrm{Ph} . \mathrm{D}$. degree for his research work in Mathematics in 2001. $\mathrm{He}$ is a faculty member of the Department of Mathematics, Jazan University, Jazan, Saudi Arabia. He is working on a fuzzy set, fuzzy group theory, fuzzy ring and fuzzy ideal theory, variational inequality, and optimization theory. E-mail: drsalah12@hotmail.com

ORCID: https://orcid.org/0000-0002-0496-3379

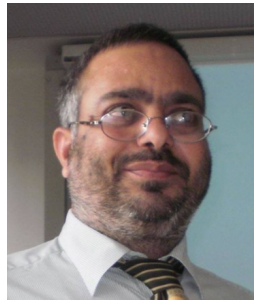

Mohammad Munir received his Ph.D. degree in Applied Mathematics from the University of Graz, Graz, Austria, in 2010. He completed his Ph.D. thesis on a project titled "Generalized Sensitivity Functions in Physiological Modelling”. His research interests are in the mathematical modelling of biological systems in the fields of the glucose-insulin dynamics, solute kinetics and hemodialysis using ordinary differential equations (ODEs). Parameter identification, sensitivity analysis and generalized sensitivity analysis are more concentrated areas of his research. His other interests include the applications of the fuzzy sets theory to multi-criteria decision-making (MCDM) problems.

Email: dr.mohammadmunir@gpgc-atd.edu.pk

ORCID: https://orcid.org/0000-0002-4891-2995 